\title{
Differentiation of Malignant and Benign Cardiac Tumors Using ${ }^{18}$ F-FDG PET/CT
}

\author{
Kambiz Rahbar ${ }^{1}$, Harald Seifarth ${ }^{2}$, Michael Schäfers ${ }^{3}$, Lars Stegger ${ }^{1}$, Andreas Hoffmeier ${ }^{4}$, Tilmann Spieker ${ }^{5}$, \\ Klaus Tiemann ${ }^{6}$, David Maintz ${ }^{2}$, Hans Heinrich Scheld ${ }^{4}$, Otmar Schober ${ }^{1}$, and Matthias Weckesser ${ }^{1}$ \\ ${ }^{1}$ Department of Nuclear Medicine, University of Münster, Münster, Germany; ${ }^{2}$ Department of Clinical Radiology, University of \\ Münster, Münster, Germany; ${ }^{3}$ European Institute for Molecular Imaging, University of Münster, Münster, Germany; ${ }^{4}$ Department of \\ Thoracic and Cardiovascular Surgery, University of Münster, Münster, Germany; ${ }^{5}$ Institute for Pathology, University of Münster, \\ Münster, Germany; and ${ }^{6}$ Department of Cardiology, University of Münster, Münster, Germany
}

In the diagnostic algorithm of cardiac tumors, the noninvasive determination of malignancy and metastatic spread is of major interest to stratify patients and to select and monitor therapies. In the diagnostic work-up, morphologic imaging modalities such as echocardiography or magnetic resonance tomography offer information on, for example, size, invasiveness, and vascularization. However, preoperative assessment of malignancy may be unsatisfactory. The aim of this study was to evaluate the diagnostic value of ${ }^{18} \mathrm{~F}-\mathrm{FDG}$ PET and the incremental diagnostic value of an optimized CT score in this clinical scenario. Methods: ${ }^{18} \mathrm{~F}-\mathrm{FDG}$ PET/CT scans (wholebody imaging with low-dose CT) of 24 consecutive patients with newly diagnosed cardiac tumors were analyzed (11 men, 13 women; mean age $\pm S D, 59 \pm 13 \mathrm{y}$ ). The maximum standardized uptake values $\left(S_{U} V_{\text {max }}\right)$ of the tumors were measured. Patients were divided into 2 groups: benign cardiac tumors $(n=7)$ and malignant cardiac tumors $(n=17)$ (cardiac primaries $[n=8]$ and metastases $[n=9])$. SUV $\max$ was compared between the 2 groups. Results were compared with contrast-enhanced CT, using standardized criteria of malignancy. Histology served as ground truth. Results: Mean $\mathrm{SUV}_{\max }$ was $2.8 \pm 0.6$ in benign cardiac tumors and significantly higher both in malignant primary and in secondary cardiac tumors $(8.0 \pm 2.1$ and $10.8 \pm 4.9, P<0.01)$. Malignancy was determined with a sensitivity of $100 \%$ and specificity of $86 \%$ (accuracy, 96\%), after a cutoff with high sensitivity $\left(\mathrm{SUV}_{\max }\right.$ of 3.5) was chosen to avoid false-negatives. Morphologic imaging reached a sensitivity of $82 \%$ and a specificity of $86 \%$ (accuracy, 83\%). Both false-positive and false-negative decisions in morphology could be corrected in all but 1 case

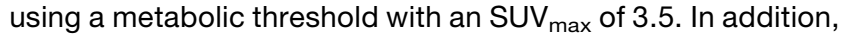
extracardiac tumor manifestations were detected in 4 patients by whole-body ${ }^{18}$ F-FDG PET/CT. Conclusion: ${ }^{18}$ F-FDG PET/ CT can aid the noninvasive preoperative determination of malignancy and may be helpful in detecting metastases of malignant cardiac tumors.

Received Jul. 18, 2011; revision accepted Jan. 24, 2012.

For correspondence or reprints contact: Kambiz Rahbar, Department of Nuclear Medicine, University Hospital Münster, Albert-Schweitzer-Campus 1, 48149 Münster, Germany.

E-mail: rahbar@uni-muenster.de

Published online May 10, 2012.

COPYRIGHT $\odot 2012$ by the Society of Nuclear Medicine, Inc.
Key Words: cardiac tumors; ${ }^{18} \mathrm{~F}-\mathrm{FDG}$ PET/CT; cardiac angiosarcoma; cardiac metastases

J Nucl Med 2012; 53:856-863

DOI: 10.2967/jnumed.111.095364

$\mathbf{P}$ ing from $0.001 \%$ to $0.028 \%$ in autopsy reports. Approximately $20 \%-25 \%$ of these tumors are malignant, with angiosarcoma being the most common malignant cardiac tumor (1-3). In contrast, secondary cardiac tumors, such as metastasis to the heart from other malignant tumors, occur more frequently (4).

Because benign tumors can be resected completely in most cases, it is important to preoperatively differentiate between benign and malignant masses. This differentiation is essential, because most tumors are not suited for catheter-based biopsy. CT and MRI can depict several morphologic features that can be used to differentiate benign from malignant tumors and, in some cases, even provide a specific diagnosis (5-9). However, today, there is no routinely performed noninvasive imaging modality determining malignancy of cardiac tumors with sufficient accuracy. Molecular imaging methods such as ${ }^{18} \mathrm{~F}-\mathrm{FDG}$ with PET can visualize tumor metabolism and thereby assess metabolic activity. PET, nowadays combined with CT into PET/CT, is a clinically established method to characterize tumors $(10,11)$.

The use of ${ }^{18} \mathrm{~F}-\mathrm{FDG}$ PET/CT to assess cardiac tumors is infrequently reported as single cases; the results of these reports are summarized in Table 1 (12-25). To date, larger patient groups undergoing whole-body ${ }^{18} \mathrm{~F}-\mathrm{FDG} \mathrm{PET} / \mathrm{CT}$ to elucidate the diagnostic performance of PET/CT in cardiac tumors have not yet been published.

Therefore, the aim of this study was to evaluate the diagnostic value of ${ }^{18} \mathrm{~F}$-FDG $\mathrm{PET} / \mathrm{CT}$ with respect to preoperatively differentiating benign from malignant cardiac tumors. 
TABLE 1

Published Cases of Cardiac Tumors and ${ }^{18}$ F-FDG PET

\begin{tabular}{|c|c|c|c|c|c|}
\hline \multirow[b]{2}{*}{ Study } & \multicolumn{3}{|c|}{ Histology } & \multirow[b]{2}{*}{${ }^{18} \mathrm{~F}-\mathrm{FDG}$} & \multirow[b]{2}{*}{$\mathrm{SUV}_{\max }$} \\
\hline & $\begin{array}{c}\text { Primary benign } \\
\text { tumors }\end{array}$ & $\begin{array}{c}\text { Primary malignant } \\
\text { tumors }\end{array}$ & $\begin{array}{c}\text { Secondary malignant } \\
\text { tumors }\end{array}$ & & \\
\hline Agostini et al. (12) & Myxoma & & & + & NA \\
\hline Freudenberg et al. (13) & & Angiosarcoma & & + & 7.5 \\
\hline Hoffmeier et al. (15) & & Angiosarcoma & & + & 5.4 \\
\hline Hori et al. (16) & & Angiosarcoma & & + & 9.9 \\
\hline Zhang et al. (22) & & Liposarcoma & & + & 5.6 \\
\hline Nakamura-Horigome et al. (17) & & Angiosarcoma & & + & NA \\
\hline Higashiyama et al. (14) & & Angiosarcoma & & + & 5.6 \\
\hline Schnabel et al. (20) & & & Metastasis & - & NA \\
\hline Plutchok et al. (19) & & & Metastasis & + & NA \\
\hline Shimotsu et al. (21) & & & Metastasis & + & 7.9 \\
\hline Biancheri et al. (23) & & & Metastasis & + & NA \\
\hline Nonaka et al. (24) & & & Metastasis & + & NA \\
\hline Orcurto et al. (18) & & & Metastasis & + & NA \\
\hline Wenning et al. (25) & & & Metastasis & + & 15.5 \\
\hline
\end{tabular}

Primary and secondary malignant cardiac tumors show increased ${ }^{18} \mathrm{~F}-\mathrm{FDG}$ uptake. In only 1 case of adenocarcinoma was ${ }^{18} \mathrm{~F}-\mathrm{FDG}$ uptake absent.

$+=$ positive; $\mathrm{NA}=$ not available; $-=$ negative.

\section{MATERIALS AND METHODS}

\section{Patients}

Twenty-four patients (11 men, 13 women; mean age \pm SD, $59 \pm 13$ y) with newly diagnosed cardiac tumors routinely undergoing ${ }^{18}$ F-FDG PET/CT (whole-body imaging with low-dose CT) as part of the initial assessment were retrospectively enrolled in this study. All patients were referred before treatment between 2004 and 2010 to screen for extracardiac tumor manifestations and to assess metabolism of the cardiac tumors.

Four of the 24 patients had a history of noncardiac tumors (time to cardiac tumor, $12.3 \pm 8.1 \mathrm{y}$ ), which had been effectively treated in all cases.

On the basis of the histologic characterization of the surgically resected cardiac tumors $(n=16)$ or tumor biopsies $(n=8)$, patients were divided into 3 groups: benign primary cardiac tumors $(n=7)$, malignant primary cardiac tumors $(n=8)$, and secondary malignant cardiac tumors (metastases of other tumors or lymphoma involvement of the heart, $n=9$ ). ${ }^{18}$ F-FDG uptake was compared among groups. For the purpose of calculating sensitivity and specificity, all malignant cardiac tumors were regarded as 1 group (Table 2).

The present study was performed according to local institutional guidelines and was approved by the ethics committee. Informed consent was obtained from all patients for the PET/CT procedure.

\section{Patient Preparation and PET/CT}

Details concerning the PET/CT procedures have been published elsewhere $(26,27)$. To optimally suppress glucose uptake in the myocardium, which could conceal uptake of the cardiac tumors, all patients were studied after fasting for at least $6 \mathrm{~h}$. Blood glucose levels at the time of ${ }^{18} \mathrm{~F}-\mathrm{FDG}$ application were less than 120 $\mathrm{mg} / \mathrm{dL}$. A body-weight-adapted activity of ${ }^{18} \mathrm{~F}-\mathrm{FDG}(5 \mathrm{MBq} / \mathrm{kg}$ of body weight) was injected intravenously (median, $68 \mathrm{~min}$; range, 54-108 min) before PET data acquisition. All patients were asked to void their bladder immediately before scanning. The scans were obtained using a hybrid PET/CT system (Biograph Sensation 16; Siemens Medical Solutions). Low-dose CT of the entire area covered by PET (from skull base to the mid thigh level) was performed for attenuation correction in all patients. Simultaneous contrast-enhanced CT on the PET/CT scanner was acquired for 7 patients (29\%). After completion of the CT scan, PET data were acquired for 3 min per bed position. PET images were reconstructed using the standard manufacturer-supplied software.

A separately acquired contrast-enhanced CT scan was available for 17 patients (71\%). CT scans were obtained either on a 16-slice or on a 64-slice scanner (Somatom Sensation 16 or Somatom Definition; Siemens Medical Solutions), with a slice thickness of 1.0 or $0.75 \mathrm{~mm}$. MRI was performed for 11 patients $(46 \%$, data not shown).

\section{Image Analysis}

Morphologic tumor characteristics were classified using CT by 2 board-certified radiologists. In addition to the size of the mass, the following $7 \mathrm{CT}$ criteria of malignancy were systematically evaluated: contrast uptake, infiltration of the tumor into the surrounding tissue, infiltration of the tumor into the epicardium, irregular tumor margin, presence of necrosis, presence of pericardial effusion, and tumor involving more than 1 chamber. If 3 or more of these features were present, a mass was classified as malignant (Table 2).

${ }^{18}$ F-FDG uptake in all cardiac tumors was quantified by calculating the maximum standardized uptake values $\left(\mathrm{SUV}_{\max }\right)$ from 3-dimensional volumes of interest encompassing the tumor mass, equaling the decay-corrected regional radioactivity divided by the total injected dose and the body weight. In the case of low ${ }^{18} \mathrm{~F}$ FDG uptake, CT images were used to identify the location and to set the volumes of interest. $\mathrm{SUV}_{\max }$ was correlated to the final diagnosis obtained by histology (Table 2). 
TABLE 2

Diagnostic Performance of Morphologic CT Features

\begin{tabular}{|c|c|c|c|c|}
\hline Feature & Sensitivity & Specificity & Positive predictive value & Negative predictive value \\
\hline Contrast enhancement & $100 \%$ & $0 \%$ & $71 \%$ & NA \\
\hline Infiltration of surrounding tissue & $76 \%$ & $86 \%$ & $93 \%$ & $60 \%$ \\
\hline Involvement of epicardium & $65 \%$ & $86 \%$ & $92 \%$ & $50 \%$ \\
\hline Irregular margin & $82 \%$ & $57 \%$ & $82 \%$ & $57 \%$ \\
\hline Presence of necrosis & $12 \%$ & $100 \%$ & $100 \%$ & $32 \%$ \\
\hline Pericardial effusion & $41 \%$ & $86 \%$ & $88 \%$ & $38 \%$ \\
\hline Involvement of more than 1 chamber & $71 \%$ & $86 \%$ & $86 \%$ & $50 \%$ \\
\hline 3 or more features & $82 \%$ & $86 \%$ & $93 \%$ & $67 \%$ \\
\hline Metabolic data & $100 \%$ & $86 \%$ & $94 \%$ & $100 \%$ \\
\hline
\end{tabular}

Sensitivity and specificity are shown for each criterion separately and for simultaneous presence of 3 or more features.

$\mathrm{NA}=$ not available.

To describe lesion-to-background contrast and to rule out artificial activity contribution by spillover of myocardial or blood ${ }^{18} \mathrm{~F}$-FDG uptake into the tumor region, myocardial background tissue and blood-pool ${ }^{18} \mathrm{~F}$-FDG uptake were also measured for all patients (Fig. 1). Circular regions of interest were placed over the myocardial tissue next to the tumor and in the lumen of the aorta ascendens (region of interest diameter, $10 \mathrm{~mm}$ ). All PET/CT scans were reviewed and interpreted by 2 board-certified nuclear medicine physicians, who were aware of clinical signs and symptoms and of the tumor location but were unaware of the final diagnosis.

\section{Statistics}

Data were analyzed using standard statistical software (PASW Statistics 18; IBM). All data presented are mean value \pm SD. A Kolmogorov-Smirnov test was performed to confirm normal distribution of the data. Patient groups were compared using the 2sided Student $t$ test for unpaired data. Significance was inferred at a level of $P$ less than 0.05 . Pearson correlation analysis was used to assess a relationship between tumor size and metabolic activity.

\section{RESULTS}

\section{Glucose Uptake}

For glucose uptake, the individual $\mathrm{SUV}_{\max }$ is shown in Table 3, a graphical analysis is shown in Figure 1, and examples are shown in Figures 2-6. The 6-h fasting protocol kept ${ }^{18} \mathrm{~F}$-FDG uptake low in the myocardium (mean standardized uptake value [SUV], $2.1 \pm 0.6$ ) and blood pool (mean SUV, $1.6 \pm 0.4$ ) in all patients, enabling delineation of hypermetabolic tumors from the myocardium (Fig. 3).

The mean ${ }^{18}$ F-FDG uptake (SUV) of the liver was $2.2 \pm$ 0.6. In the 24 cardiac tumors, a 3- to 4-fold uptake was found (mean $\mathrm{SUV}_{\max }, 7.5 \pm 3.7$ ). However, the range of uptake between tumor entities was large, from virtually no uptake to highly increased glucose uptake $\left(\mathrm{SUV}_{\max }, 1.6-\right.$ 16.7). Against background in the myocardium or blood, 16 of 24 tumors $(66 \%)$ showed a positive contrast. There was
FIGURE 1. Glucose uptake as quantified by ${ }^{18} \mathrm{~F}-\mathrm{FDG}$ PET (SUV) in various cardiac tumors, myocardium, and blood. Uptake is consistently low in myocardium and blood. Malignant primary cardiac tumors show significantly higher glucose uptake than benign primary tumors. Malignant secondary cardiac tumors show mean glucose uptake comparable to that of malignant primary tumors; however, range is considerably larger.

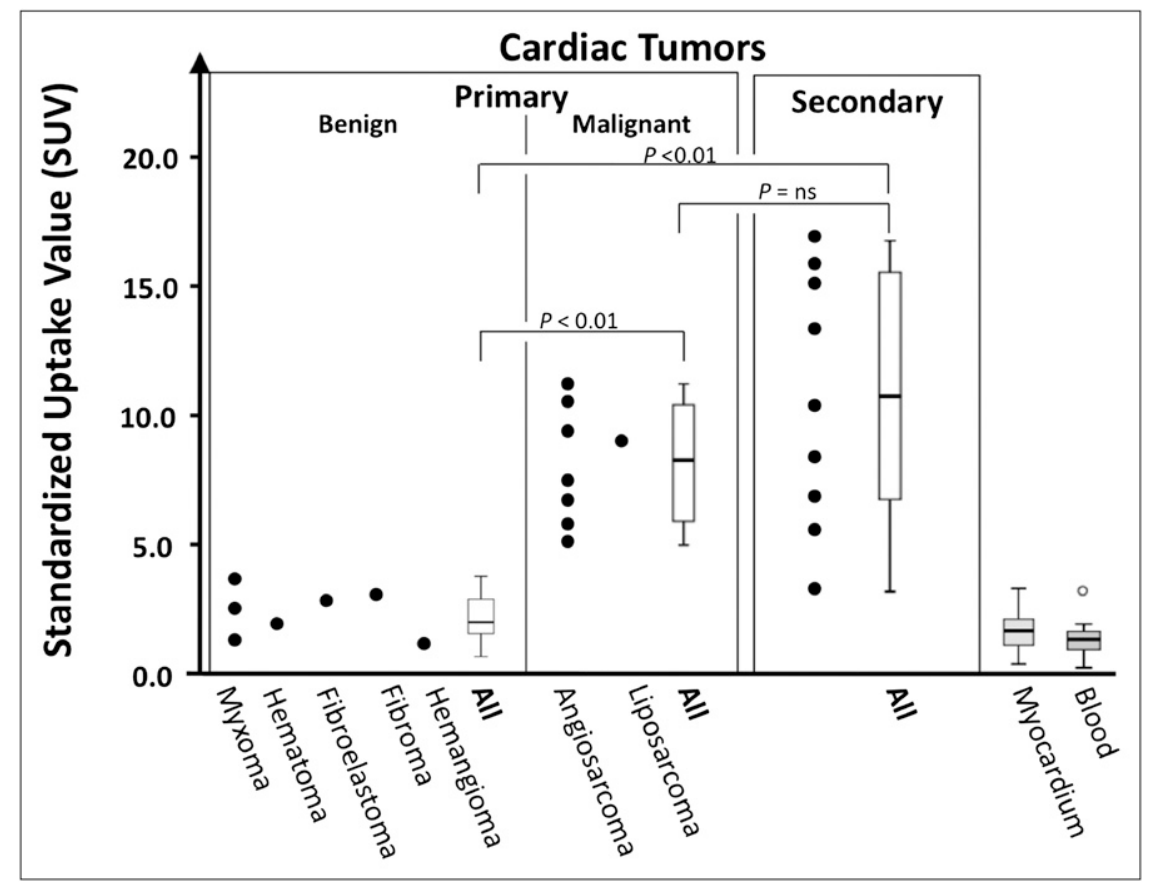




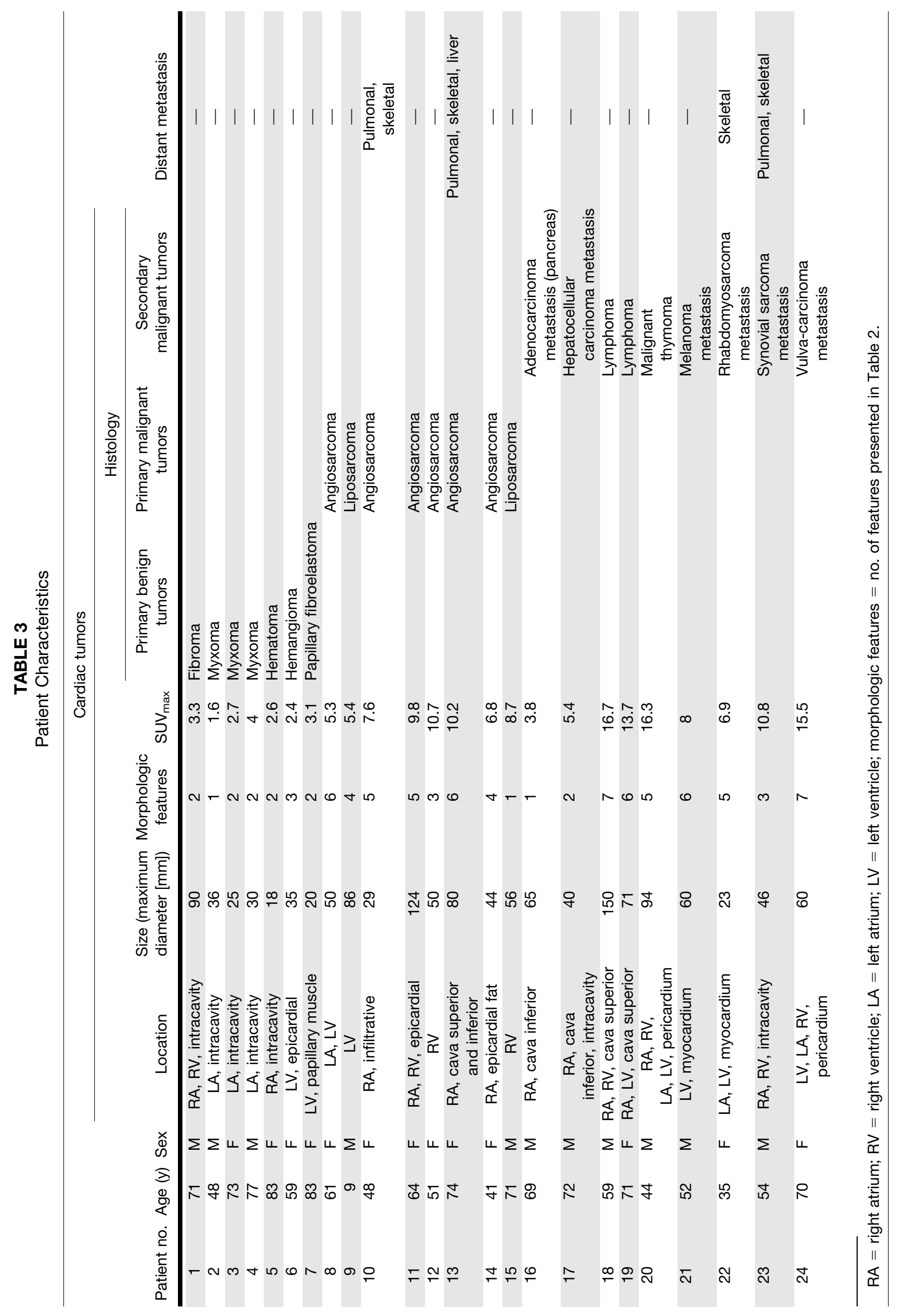




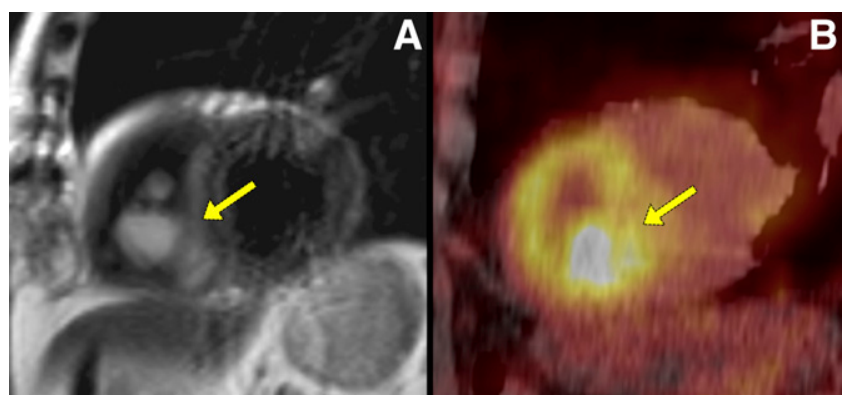

FIGURE 2. A 71-y-old man presenting with liposarcoma in right ventricle. ${ }^{18} \mathrm{~F}$-FDG PET/CT images show increased ${ }^{18} \mathrm{~F}$-FDG-uptake in tumor ( $B$, arrow). T2-weighted MR images show hyperintense tumor in right ventricle attached to septum (A, arrow).

a significant correlation between tumor size and $\mathrm{SUV}_{\max }$ $\left(R^{2}=0.42\right)$. Tumor size as a diagnostic parameter for malignancy could not differ between benign and malignant tumors $(P=0.33)$.

In the 7 patients with benign primary cardiac tumors, glucose uptake was low, with a mean $\mathrm{SUV}_{\max }$ of $2.8 \pm 0.6$; these tumors typically did not exhibit a positive contrast to the myocardium and were visible on morphologic images only (Fig. 4).

In contrast, the 8 patients with malignant primary cardiac tumors (Fig. 5) showed an approximately 2.5 times higher mean $\mathrm{SUV}_{\text {max }}$ than did those patients in the benign cardiac tumor group (Fig. $1 ; P<0.001$ ). Among the 8 patients with malignant primary cardiac tumors, there were 2 cases of skeletal metastases (25\%); both patients had additional lung metastases and 1 had an additional liver metastasis. All cardiac primaries with metastases were angiosarcomas.

In the 9 cases with secondary cardiac tumors (Fig. 6) resulting from metastases, a mean $\mathrm{SUV}_{\max }$ of $10.8 \pm 4.9$ was found, with a relatively wide range of ${ }^{18} \mathrm{~F}-\mathrm{FDG}$ uptake (range, 3.4-16.7). ${ }^{18} \mathrm{~F}-\mathrm{FDG}$ uptake in secondary cardiac tumors was significantly higher than that in benign primary cardiac tumors $(P=0.001)$ and similar to that of malignant primary tumors $(P=$ not statistically significant). In 2 of the 9 cases, previously unsuspected metastases were detected by ${ }^{18}$ F-FDG PET: skeletal metastases and an additional lung metastasis.

To evaluate the best cutoff value, a receiver-operatingcharacteristic analysis was performed. According to this analysis, 2 different cutoff values can be proposed: 3.5 and 4.6. An $\mathrm{SUV}_{\max }$ of 3.5 reveals a sensitivity of $100 \%$ and specificity of $86 \%$, with a positive predictive value of $94 \%$ and a negative predictive value of $100 \%$. With an $\mathrm{SUV}_{\max }$ of 4.6 , the sensitivity drops to $94 \%$ and the specificity rises to $100 \%$, with a positive predictive value of $100 \%$.

\section{CT}

The diagnostic performance of the 7 morphologic characteristics and their combination is shown in Tables 2 and 3. All tumors showed contrast enhancement on CT. Fourteen of 17 malignant lesions were correctly classified with $\mathrm{CT}$ using the morphologic score (i.e., presence of 3 or

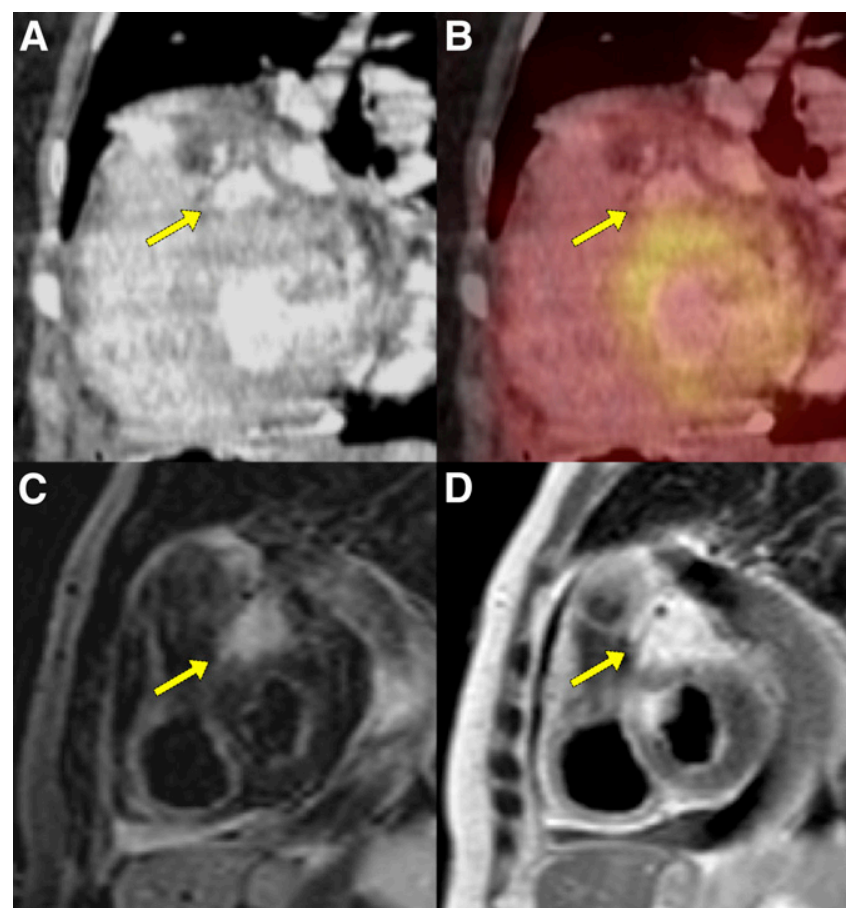

FIGURE 3. A 59-y-old woman presenting with pleural, pericardial effusion and chest pain. Cardiac CT revealed left ventricular epicardial tumor (A, arrow). MR images show hypervascular epicardial tumor (C, T2 hyperintense; D, contrast-enhanced T1) and large pericardial effusion. ${ }^{18} \mathrm{~F}-\mathrm{FDG}$ PET/CT revealed low uptake within tumor (B, arrow).

more criteria; sensitivity, $82 \%$ ) and only 1 benign lesion was misclassified as malignant (specificity, $85 \%$ ). The 3 misclassified malignant lesions were 2 metastases (1 hepatocellular carcinoma and 1 adenocarcinoma arising from the pancreas) that protruded into the right atrium from the

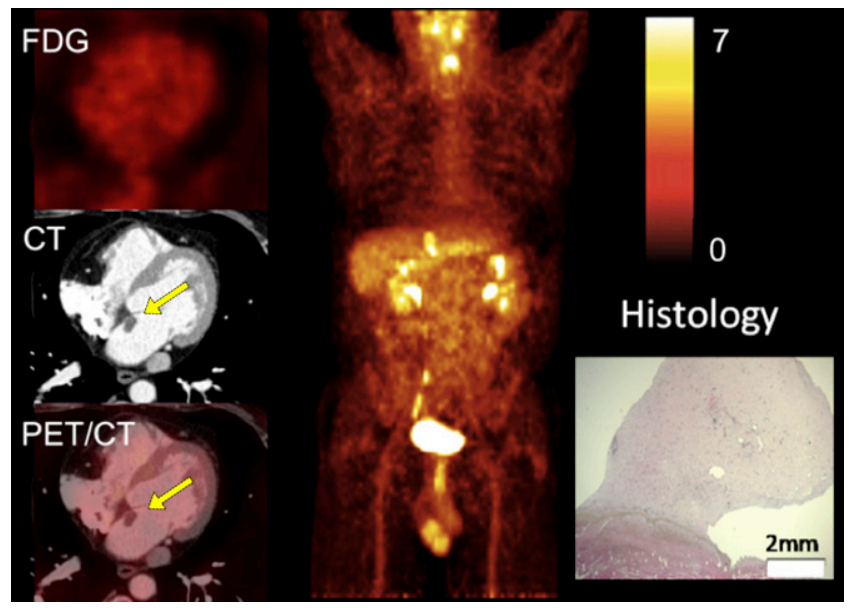

FIGURE 4. A 48-y-old man presenting with incidentally diagnosed cardiac mass in left atrium on echocardiography: transaxial slices zoomed to heart (left), maximum-intensity projection of trunk (middle), and histology image (right). SUV max $_{\text {of cardiac tumor (yellow }}$ arrows) was not increased against background, and there were no further ${ }^{18} \mathrm{~F}-\mathrm{FDG}$-positive lesions throughout whole-body ${ }^{18} \mathrm{~F}-\mathrm{FDG}$ PET/CT. Histologic work-up after tumor resection revealed benign primary cardiac myxoma. 


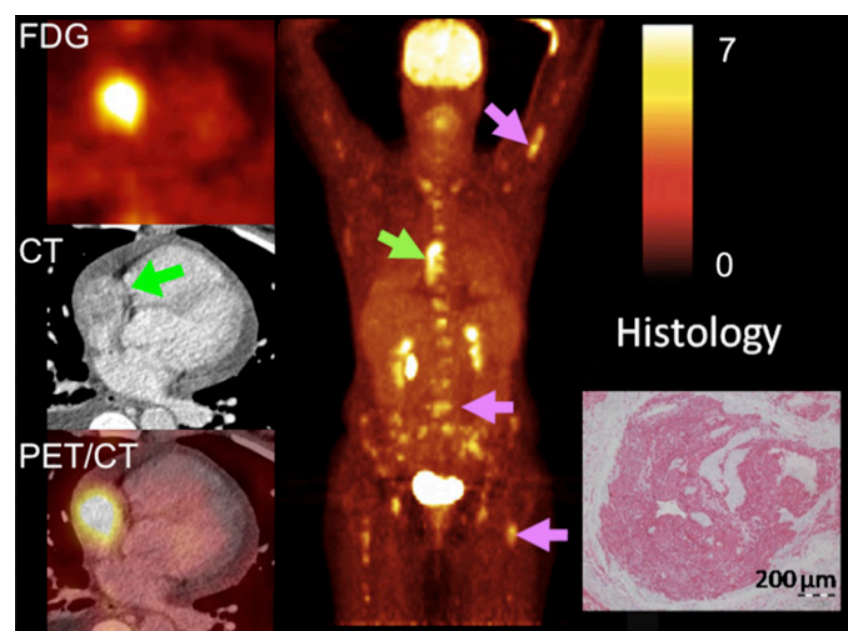

FIGURE 5. A 48-y-old woman initially presenting with dyspnea and pleural effusion: transaxial slices zoomed to heart (left), maximum-intensity projection of trunk (middle), and histology image (right). Thorax CT revealed right atrial tumor mass, which on PET showed strong ${ }^{18} \mathrm{~F}-\mathrm{FDG}$ uptake (green arrows). Whole-body ${ }^{18} \mathrm{~F}-$ FDG PET/CT assessed diffuse bone marrow metastases (pink arrows, right). Histologic work-up of tumor biopsy revealed malignant primary cardiac tumor and angiosarcoma.

inferior vena cava. The third misclassified lesion was a liposarcoma that involved only the right ventricle and showed only a broad attachment to the right ventricular wall but no clear sign of infiltration (Fig. 2). The misclassified benign lesion was a hemangioma situated in the epicardial fat next to the origin of the left coronary artery, which showed 3 features otherwise used to classify malignant tumors-namely, contrast enhancement, involvement of the epicardial fat, and pericardial effusion (Fig. 3). The receiver-operating-characteristic analysis

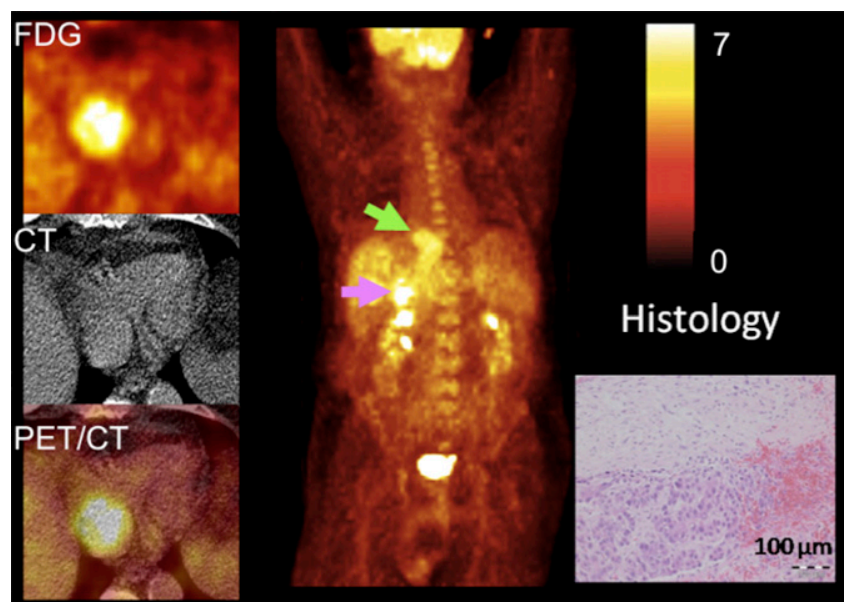

FIGURE 6. A 72-y-old man presenting with chest pain, for which right atrial tumor was diagnosed by cardiac MRI: transaxial slices zoomed to heart (left), maximum-intensity projection of trunk (middle), and histology image (right). ${ }^{18} \mathrm{~F}-\mathrm{FDG} \mathrm{PET} / \mathrm{CT}$ revealed strong ${ }^{18} \mathrm{~F}-\mathrm{FDG}$ uptake of cardiac tumor (green arrows); additional abnormal ${ }^{18} \mathrm{~F}-\mathrm{FDG}$ uptake was recognized in liver (pink arrow, right). Histologic work-up revealed hepatocellular carcinoma and distant metastasis to heart. revealed an optimal cutoff of 3 morphologic criteria, with a high specificity of $100 \%$ and a sensitivity of $70 \%$. Using a threshold of malignancy of 4 or more morphologic criteria increased the positive predictive value to $100 \%$ at the cost of a lower sensitivity of $71 \%$.

\section{DISCUSSION}

${ }^{18}$ F-FDG uptake reflects the metabolic rate of glycolysis in tumors and thus supplies additional information to morphologic imaging. Today, ${ }^{18} \mathrm{~F}$-FDG PET has grown to be an important tool in the diagnostic management of patients with a variety of malignant tumors $(10,11,28-31)$.

Overall, there is a correlation between the glucose accumulation in tumor tissue and the presence of malignancy, although exceptions are known $(11,29,30,32,33)$. However, ${ }^{18} \mathrm{~F}-\mathrm{FDG}$ PET has not yet been systematically evaluated for the characterization of cardiac tumors.

Contrast-enhanced CT visualizes several morphologic features that can help to differentiate benign from malignant tumors. In a series from Hoffmann et al., a location of the tumor outside the left heart, tissue inhomogeneity, and contrast enhancement were highly sensitive markers for malignancy, but the specificity of these features was rather low. On the other hand, the presence of a pericardial effusion was highly specific for malignant tumors (8). Similar to our study, tumor size was neither sensitive nor specific for ruling out malignancy. Other authors suggested that a well-defined tumor margin and the absence of tumor infiltration into adjacent structures are predictors for benign tumors (9), whereas infiltrative growth and lobulated margins were associated with malignancy (6). CT is currently recommended, especially for the evaluation of extracardiac masses that infiltrate into the pericardium or the heart itself (34) and to detect calcification (5); however, the extent of infiltration into the myocardium can best be appreciated in MRI. For both MRI and CT, several features have been described to differentiate benign from malignant lesions $(34,35)$. However, because neither feature alone is efficient to differentiate benign from malignant lesions, we established a morphologic score that was helpful to differentiate benign from malignant lesions. Malignant tumors could be differentiated from benign lesions, with a sensitivity of $82 \%$ and a specificity of $86 \%$, using this score. The score was created primarily for $\mathrm{CT}$; therefore, signal intensities were not considered.

The present study shows that quantification of ${ }^{18} \mathrm{~F}-\mathrm{FDG}$ uptake can support the noninvasive, pretreatment differentiation between benign and malignant cardiac tumors. The published cases of ${ }^{18} \mathrm{~F}$-FDG PET uptake in cardiac tumors are in line with the results of the present study: malignant cardiac tumors typically exhibit a high ${ }^{18}$ F-FDG uptake. $\mathrm{SUV}_{\max }$ was available in 7 cardiac malignancies only (mean $\pm \mathrm{SD}, 8.8 \pm 3.63 ; 9.5 \pm 4.0$ in the present study) $(13-16,21,22,25)$. Benign cardiac tumors are expected to show only slight ${ }^{18} \mathrm{~F}-\mathrm{FDG}$ uptake, although an exception was 
reported of a myxoma presenting with high ${ }^{18} \mathrm{~F}-\mathrm{FDG}$ uptake (12). Consistently, we found an $\mathrm{SUV}_{\max }$ of $2.8 \pm 0.9$, which was in the range of background myocardial and blood activity.

With a cutoff $\mathrm{SUV}_{\max }$ of $3.5,{ }^{18} \mathrm{~F}$-FDG PET could be used to noninvasively determine tumor malignancies with a sensitivity of $100 \%$ in our series. Only 1 benign tumor was misdiagnosed (specificity, 86\%). Larger studies should further define the optimal cutoff.

Positive predictive value was $100 \%$ using a cutoff of 4 or more morphologic criteria. In these cases, PET is not necessary to evaluate for malignancy (12/24 in the present series). In patients with 3 or fewer morphologic criteria, PET was falsepositive in 1 patient only. Twenty-three of 24 patients were correctly diagnosed (accuracy, 96\%) and negative predictive value was $100 \%$ using the combined algorithm.

The main limitation of this study is its retrospective nature and the fact that no standard diagnostic pathway existed for patients presenting with cardiac tumors, resulting in heterogeneous data regarding the availability of CT and MRI. In the present series, most of the PET/CT scans were obtained for patients in whom other imaging modalities did not securely rule out malignancy. Because some benign lesions, especially myxomas, can be diagnosed with either echocardiography or MRI, these patients were not included in this retrospective analysis. Because the distribution of benign and malignant tumors does not reflect the incidence, a referral bias must be assumed.

Furthermore, uptake time, blood glucose levels, insulin medication, and equipment affect SUV measurements. Therefore, the SUV quantified in this case series may not reliably distinguish benign from malignant cardiac tumors in other institutions, using different scanning procedures. The diagnostic algorithm we suggest is valid only for this series and has to be evaluated in larger trials.

Finding a weak correlation between the size of tumors and the $\mathrm{SUV}_{\max }$ is at least partially due to the partial-volume effect. In the setting of cardiac tumors, the partialvolume effect originates from limited scanner resolution and from tumor movement introduced both by cardiac motion and by breathing.

The accuracy of fusion of the metabo-morphologic information may be greatly enhanced by correction of both PET and CT for respiratory and cardiac motion - not yet a standard procedure in clinical PET/CT $(36,37)$.

For future studies, we propose a multicenter design including gated ${ }^{18} \mathrm{~F}$-FDG PET/CT using the diagnostic criteria and cutoffs presented here. There may still be the need to optimize CT criteria, in particular to assess whether the number of criteria can be reduced to improve accuracy. The limited patient number excluded multivariate analysis of this issue.

\section{CONCLUSION}

In selected cases, ${ }^{18} \mathrm{~F}-\mathrm{FDG} \mathrm{PET} / \mathrm{CT}$ provides incremental diagnostic information in the determination of malignancy and in staging. ${ }^{18} \mathrm{~F}-\mathrm{FDG}$ PET/CT may gain importance in the therapeutic management of patients with cardiac tumors. Future prospective studies in larger patient groups are desirable to establish an optimal imaging pathway before therapy.

\section{DISCLOSURE STATEMENT}

The costs of publication of this article were defrayed in part by the payment of page charges. Therefore, and solely to indicate this fact, this article is hereby marked "advertisement" in accordance with 18 USC section 1734.

\section{ACKNOWLEDGMENTS}

We thank the PET radiochemistry group at the Department of Nuclear Medicine for highly reliable production of ${ }^{18} \mathrm{~F}-\mathrm{FDG}$ and the radiographers at the PET/CT for excellent technical assistance. This study was supported in part by the Sonderforschungsbereich SFB 656, Molecular Cardiovascular Imaging, Münster, Germany (projects C2 and C6), and the German Research Foundation (DFG) projects HO 4295/1-1 and SP 1249/2-1. No other potential conflict of interest relevant to this article was reported.

\section{REFERENCES}

1. Burke A, Virmani S. Atlas of Tumor Pathology. Washington, DC: WashingtonArmed Force Institute of Tumor Pathology; 1996.

2. Reynen K. Cardiac myxomas. N Engl J Med. 1995;333:1610-1617.

3. Bussani R, De-Giorgio F, Abbate A, Silvestri F. Cardiac metastases. J Clin Pathol. 2007;60:27-34.

4. Chiles C, Woodard PK, Gutierrez FR, Link KM. Metastatic involvement of the heart and pericardium: CT and MR imaging. Radiographics. 2001;21:439-449.

5. Hoey ET, Mankad K, Puppala S, Gopalan D, Sivananthan MU. MRI and CT appearances of cardiac tumours in adults. Clin Radiol. 2009;64:1214-1230.

6. Araoz PA, Eklund HE, Welch TJ, Breen JF. CT and MR imaging of primary cardiac malignancies. Radiographics. 1999;19:1421-1434.

7. Castillo JG, Silvay G. Characterization and management of cardiac tumors. Semin Cardiothorac Vasc Anesth. 2010;14:6-20.

8. Hoffmann U, Globits S, Schima W, et al. Usefulness of magnetic resonance imaging of cardiac and paracardiac masses. Am J Cardiol. 2003;92:890-895.

9. Araoz PA, Mulvagh SL, Tazelaar HD, Julsrud PR, Breen JF. CT and MR imaging of benign primary cardiac neoplasms with echocardiographic correlation. Radiographics. 2000;20:1303-1319.

10. Weber WA. Assessing tumor response to therapy. J Nucl Med. 2009;50(suppl 1):1S-10S.

11. Weber WA. Positron emission tomography as an imaging biomarker. J Clin Oncol. 2006;24:3282-3292.

12. Agostini D, Babatasi G, Galateau F, Grollier G, Potier JC, Bouvard G. Detection of cardiac myxoma by F-18 FDG PET. Clin Nucl Med. 1999;24:159-160.

13. Freudenberg LS, Rosenbaum SJ, Schulte-Herbruggen J, et al. Diagnosis of a cardiac angiosarcoma by fluorine-18 fluordeoxyglucose positron emission tomography. Eur Radiol. 2002;12(suppl 3):S158-S161.

14. Higashiyama S, Kawabe J, Hayashi T, et al. Effectiveness of preoperative PET examination of huge angiosarcoma of the heart. Clin Nucl Med. 2009;34:99-102.

15. Hoffmeier A, Etz C, Schmid C, et al. Images in cardiovascular medicine: cardiac transplantation for giant sarcoma of the left ventricle. Circulation. 2005;112: e247-e249.

16. Hori Y, Funabashi N, Miyauchi H, et al. Angiosarcoma in the right atria demonstrated by fusion images of multislice computed tomography and positron emission tomography using F-18 fluoro-deoxyglucose. Int $J$ Cardiol. 2007; 123:e15-e17.

17. Nakamura-Horigome M, Koyama J, Eizawa T, et al. Successful treatment of primary cardiac angiosarcoma with docetaxel and radiotherapy. Angiology. 2008;59:368-371.

18. Orcurto MV, Delaloye AB, Letovanec I, Martins Favre M, Prior JO. Detection of an asymptomatic right-ventricle cardiac metastasis from a small-cell lung cancer by F-18-FDG PET/CT. J Thorac Oncol. 2009;4: $127-130$. 
19. Plutchok JJ, Boxt LM, Weinberger J, Fawwaz R, Sherman WH, Van Heertum RL. Differentiation of cardiac tumor from thrombus by combined MRI and F-18 FDG PET imaging. Clin Nucl Med. 1998;23:324-325.

20. Schnabel R, Koniordos P, Mohr-Kahaly S, Meyer J, Kohler HH, Khaw AV. Primary adenocarcinoma of the left atrium mimicking benign myxoma. Z Kardiol. 2003;92:254-259.

21. Shimotsu Y, Ishida Y, Fukuchi K, et al. Fluorine-18-fluorodeoxyglucose PET identification of cardiac metastasis arising from uterine cervical carcinoma. J Nucl Med. 1998;39:2084-2087.

22. Zhang M, Li B, Jiang X. PET/CT imaging in a case of cardiac liposarcoma. J Nucl Cardiol. 2008;15:473-475.

23. Biancheri I, Zsigmond R, Angoue O, et al. F-18 FDG PET identification of right atrium metastasis from a vesical carcinoma. Clin Nucl Med. 2007;32: 812-815.

24. Nonaka A, Stugaard M, Ueda O, Hara H, Shimada T, Shiotani H. Fluorodeoxyglucose-positron emission tomography differentiating thrombus from tumor in the left ventricle. J Am Coll Cardiol. 2009;53:894.

25. Wenning C, Engelen MA, Rahbar K, et al. Therapy refractory coronary compression caused by a cardiac metastasis: the role of imaging. $\mathrm{J} \mathrm{Nucl} \mathrm{Cardiol}$. 2010;17:696-698.

26. Juergens KU, Oei ML, Weckesser M, et al. Whole-body imaging of oncologic patients using 16-channel PET-CT: evaluation of an i.v. contrast enhanced MDCT protocol. Nuklearmedizin. 2008;47:30-36.

27. Weckesser M, Stegger L, Juergens KU, Wormanns D, Heindel W, Schober O. Correlation between respiration-induced thoracic expansion and a shift of central structures. Eur Radiol. 2006;16:1614-1620.
28. Fletcher JW, Djulbegovic B, Soares HP, et al. Recommendations on the use of ${ }^{18}$ F-FDG PET in oncology. J Nucl Med. 2008;49:480-508.

29. Weber WA, Avril N, Schwaiger M. Relevance of positron emission tomography (PET) in oncology. Strahlenther Onkol. 1999;175:356-373.

30. Fischman AJ. Positron emission tomography in the clinical evaluation of metastatic cancer. J Clin Oncol. 1996;14:691-696.

31. Benz MR, Czernin J, Allen-Auerbach MS, et al. FDG-PET/CT imaging predicts histopathologic treatment responses after the initial cycle of neoadjuvant chemotherapy in high-grade soft-tissue sarcomas. Clin Cancer Res. 2009;15:2856-2863.

32. Reske SN, Bares R, Bull U, Guhlmann A, Moser E, Wannenmacher MF. Clinical value of positron emission tomography (PET) in oncologic questions: results of an interdisciplinary consensus conference. Schirmerreschaft der Deutschen Gesellschaft for Nuklearmedizin. Nuklearmedizin. 1996;35:42-52.

33. Reske SN, Kotzerke J. FDG-PET for clinical use: results of the 3rd German interdisciplinary consensus conference, "Onko-PET III", 21 July and 19 September 2000. Eur J Nucl Med. 2001;28:1707-1723.

34. Krombach GA, Spuentrup E, Buecker A, et al. Heart tumors: magnetic resonance imaging and multislice spiral CT. Rofo. 2005;177:1205-1218.

35. Grebenc ML, Rosado de Christenson ML, Burke AP, Green CE, Galvin JR. Primary cardiac and pericardial neoplasms: radiologic-pathologic correlation. Radiographics. 2000;20:1073-1103; quiz 1110-1111, 1112.

36. Büther F, Stegger L, Dawood M, et al. Effective methods to correct contrast agent-induced errors in PET quantification in cardiac PET/CT. J Nucl Med. 2007;48:1060-1068.

37. Dawood M, Kosters T, Fieseler M, et al. Motion correction in respiratory gated cardiac PET/CT using multi-scale optical flow. Med Image Comput Comput Assist Interv. 2008;11:155-162. 